\title{
Thermal analytical characteristics by TGA-DTA-DSC analysis of Carica papaya leaves from Kachchh
}

\author{
Vijay R. Ram ${ }^{1, *}$, Pravin N. Ram ${ }^{1}$, Taslimahemad T. Khatri ${ }^{1}$, Suhas J. Vyas ${ }^{2}$, \\ Pragnesh N. Dave ${ }^{1}$ \\ ${ }^{1}$ Department of Chemistry, KSKV Kachchh University, Bhuj - 370 001, Gujarat, India \\ ${ }^{2}$ Department of Earth and Environment Science, KSKV Kachchh University, \\ Bhuj - 370 001, Gujarat, India \\ *E-mail address: ram_vijay1982@yahoo.co.in
}

\begin{abstract}
An experimental study on Carica papaya leaves was carried out in Thermo gravimetric analyzer (TGA), Differential Thermal Analyzer (DTA) and Differential Scanning Calorimetric (DSC) analyzer to investigate the effects of reaction atmosphere on thermal chemical characteristics. Experimental results show that In DSC curve, Endothermic peak at $101{ }^{\circ} \mathrm{C}$ is attributed to dehydration/Water loss from surface and pores of the powder sample. Step at $215{ }^{\circ} \mathrm{C}$ is associated with second order phase transition such as Glass Transition and it should be further confirmed in second heating (During heatcool- heat cycle). Endothermic peak at $336{ }^{\circ} \mathrm{C}$ is associated protease thermal decomposition /Beta Cyclodextrin breakdown. In the TGA Curve, The initial $4 \%$ weight loss is due to water loss from surface/pores of powder sample. Second weight loss between $200-450{ }^{\circ} \mathrm{C}$ is associated to degradation of cellulose and hemicellulose.
\end{abstract}

Keywords: Thermal (TGA-DTA-DSC) analysis; Carica papaya leaves; Semi arid region; Glass Transition; weight loss

\section{INTRODUCTION}

Importance of the chemical constituents of plants used in ancient indian medical system. Mutalic ${ }^{[1]}$ paper on "Research Needs and Traditional Medicine in South East Asia Region" has emphasized for research in traditional medicine.

A pioneering work by Kirtikar and Basu ${ }^{[2]}$, Chopra et al ${ }^{[3]}$. And Nadkarni ${ }^{[4]}$ have led to compilation of information with regard to occurrence, identity and therapeutic properties and chemical constituents of such plants remedies particularly used in Ayurvedic and Unani system of medicine.

Carica papaya, belongs to the family of Caricaceae, and several species of Caricaceae have been used as remedy against a variety of diseases ${ }^{[5]}$. 

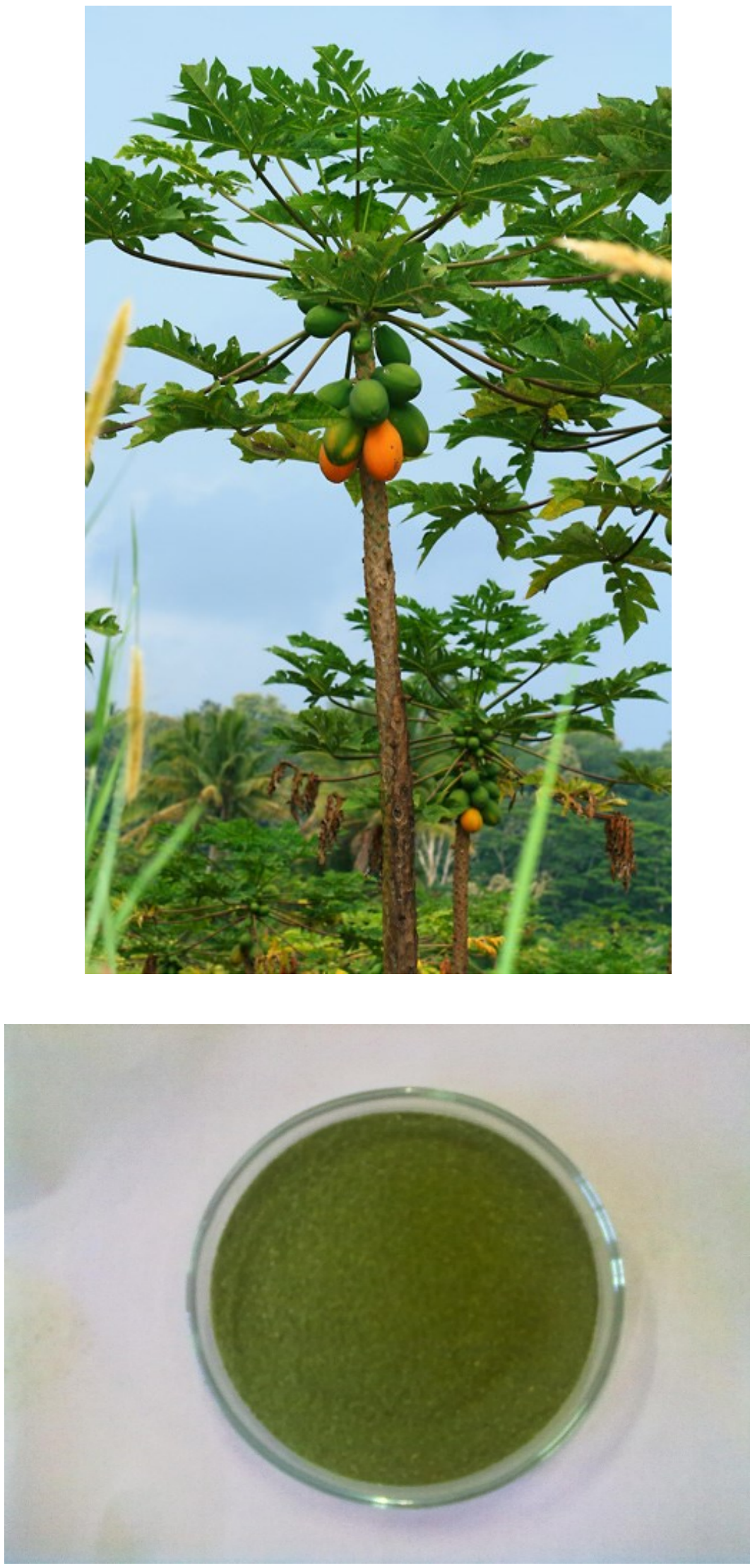

Photo 1. Plates of C. papaya growing in semi-arid region of Kachchh. 
The leaves of papaya have been shown to contain many active components that can increase the total antioxidant power in blood and reduce lipid peroxidation level, such as papain, chymopapain, cystatin, tocopherol, ascorbic acid, flavonoids, cyanogenic glucosides and glucosinolates ${ }^{[6]}$.

In spite of the concurrent use of the extract of Carica papaya with prescription oral hypoglycemic agents in some patients ${ }^{[7,8]}$ there is a dearth of literature on the effects of the extract on activity of oral hypoglycaemic agents.

Similar kind of study on the mineral composition of leaves of C. papaya was analyzed by traditional and normal methodology for mineral ${ }^{[9]}$, XRF analysis of Carica papaya leaves also reported in literature ${ }^{[10]}$.

\section{EXPERIMENTAL}

\section{1. Sample Preparation}

Leaves of $C$. papaya were collected from different habitats of Kachchh region of Gujarat during December, 2013. Leaves were wash with distilled water and sun dried, after then it was grinded in mixture powder of leaves sample were prepared and were used for further elemental analysis in TGA-DTA-DSC instrument.

\section{2. Instrumental Parameter}

Current study was carried out on Thermal Analysis Equipment (TGA, DTA, DSC), Make and Model: Perkin STA 8000. Analysis was carried out at heat from $50{ }^{\circ} \mathrm{C}$ to $500{ }^{\circ} \mathrm{C}$ at $20{ }^{\circ} \mathrm{C} / \mathrm{min}$ rate with suitable cooling attachment with thermocouple sensor $\mathrm{Pt}-\mathrm{Pt} / \mathrm{Rh}$. For the analysis sample Weight taken $7.654 \mathrm{mg}$.

\section{RESULT AND DISCUSSION}

In DSC curve, Endothermic peak at $101{ }^{\circ} \mathrm{C}$ is attributed to dehydration/Water loss from surface and pores of the powder sample. Step at $215^{\circ} \mathrm{C}$ is associated with second order phase transition such as Glass Transition and it should be further confirmed in second heating (During heat- cool- heat cycle).

Endothermic peak at $336{ }^{\circ} \mathrm{C}$ is associated protease thermal decomposition /Beta Cyclodextrin breakdown. In the TGA Curve, the initial $4 \%$ weight loss is due to water loss from surface/pores of powder sample. Second weight loss between $200-450{ }^{\circ} \mathrm{C}$ is associated to degradation of cellulose and hemicellulose.

In further analysis there is possibility of degradation of lignin between $450-800{ }^{\circ} \mathrm{C}$ by further extending the analysis temperature.

Data of thermal analysis by TGA-DTA-DSC instrument are as shown in the figures described below. 


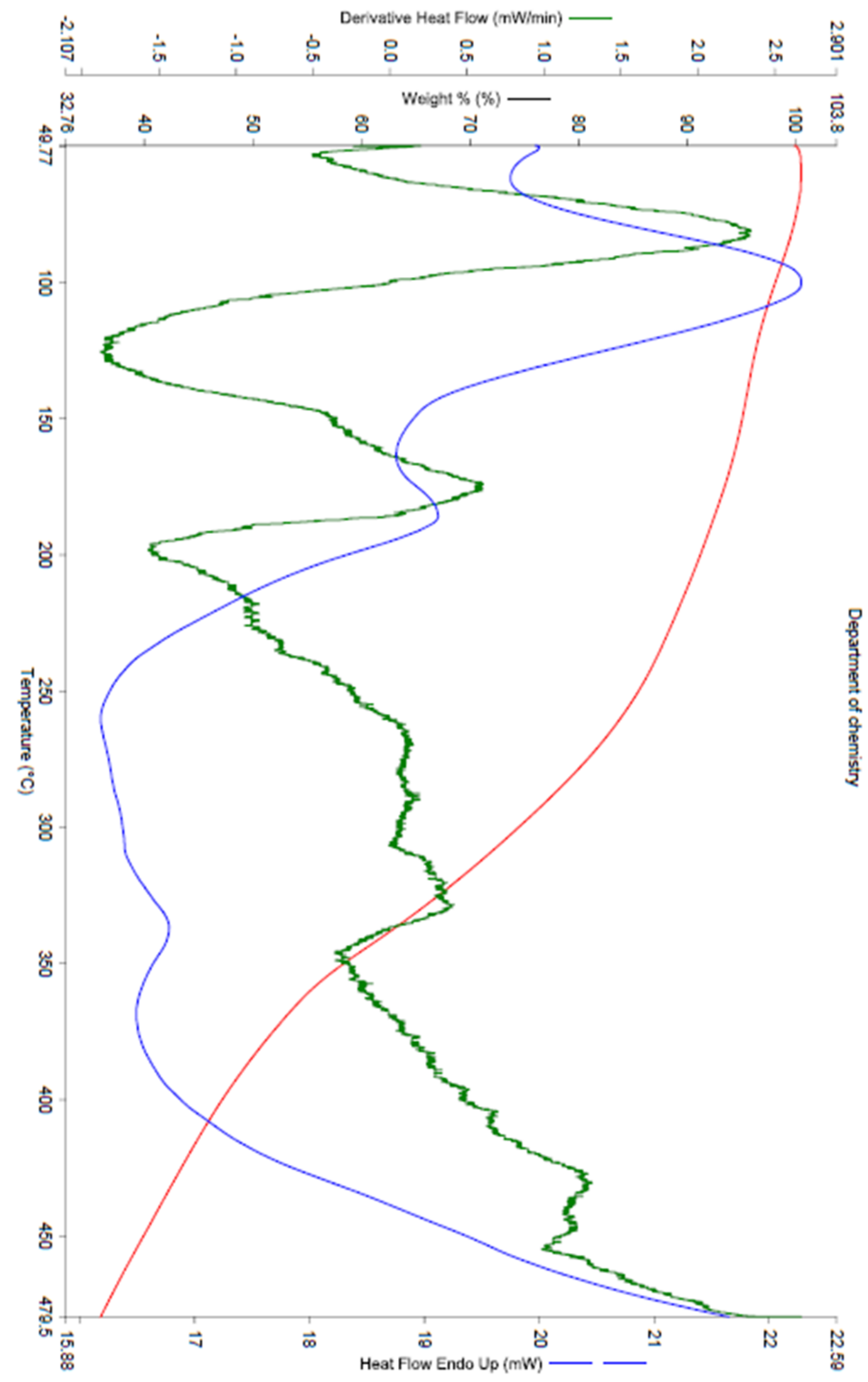

Figure 1. DSC Thermogram of the leaves of C. papaya. 


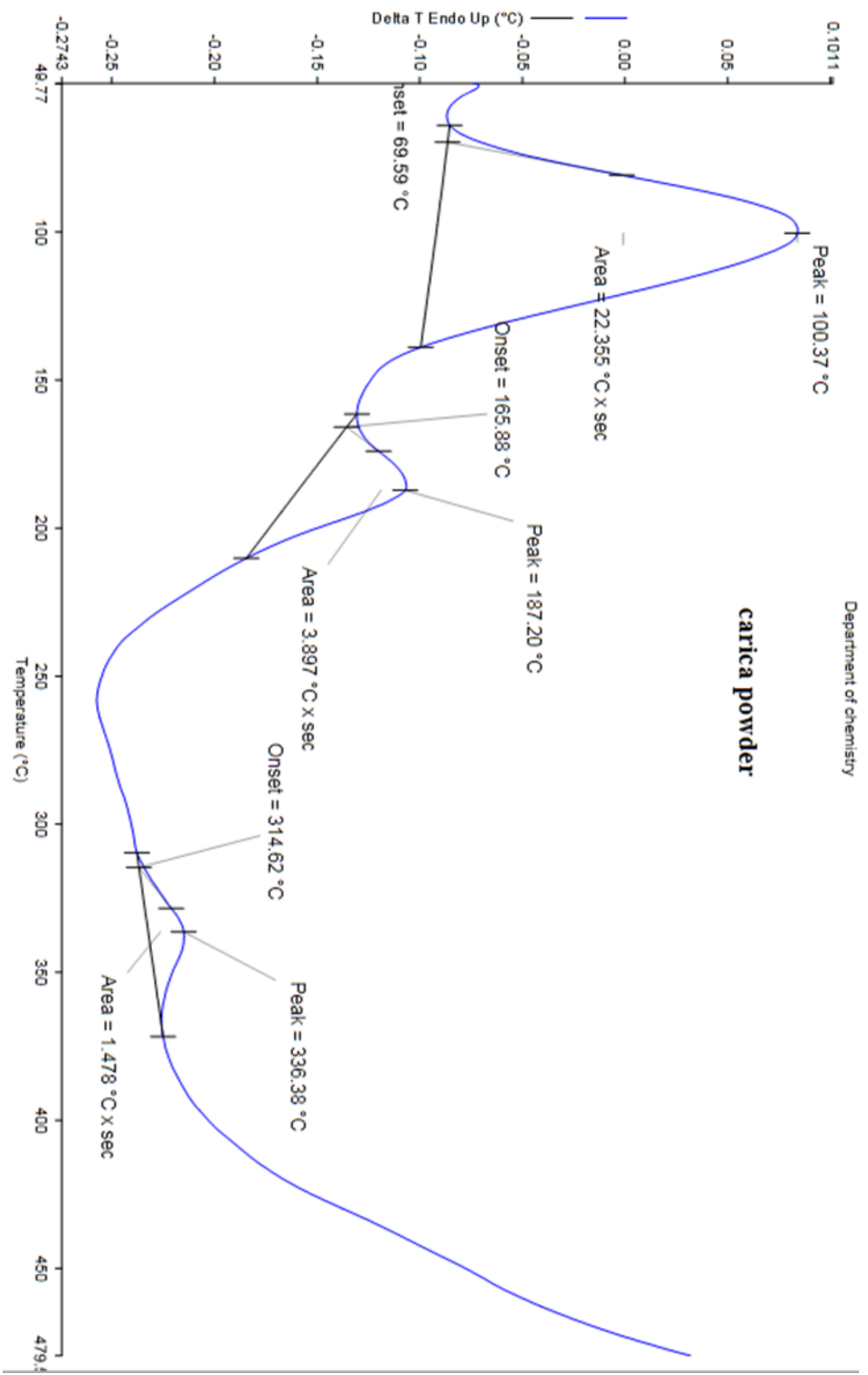

Figure 2. DTA Thermogram of the leaves of C. papaya. 


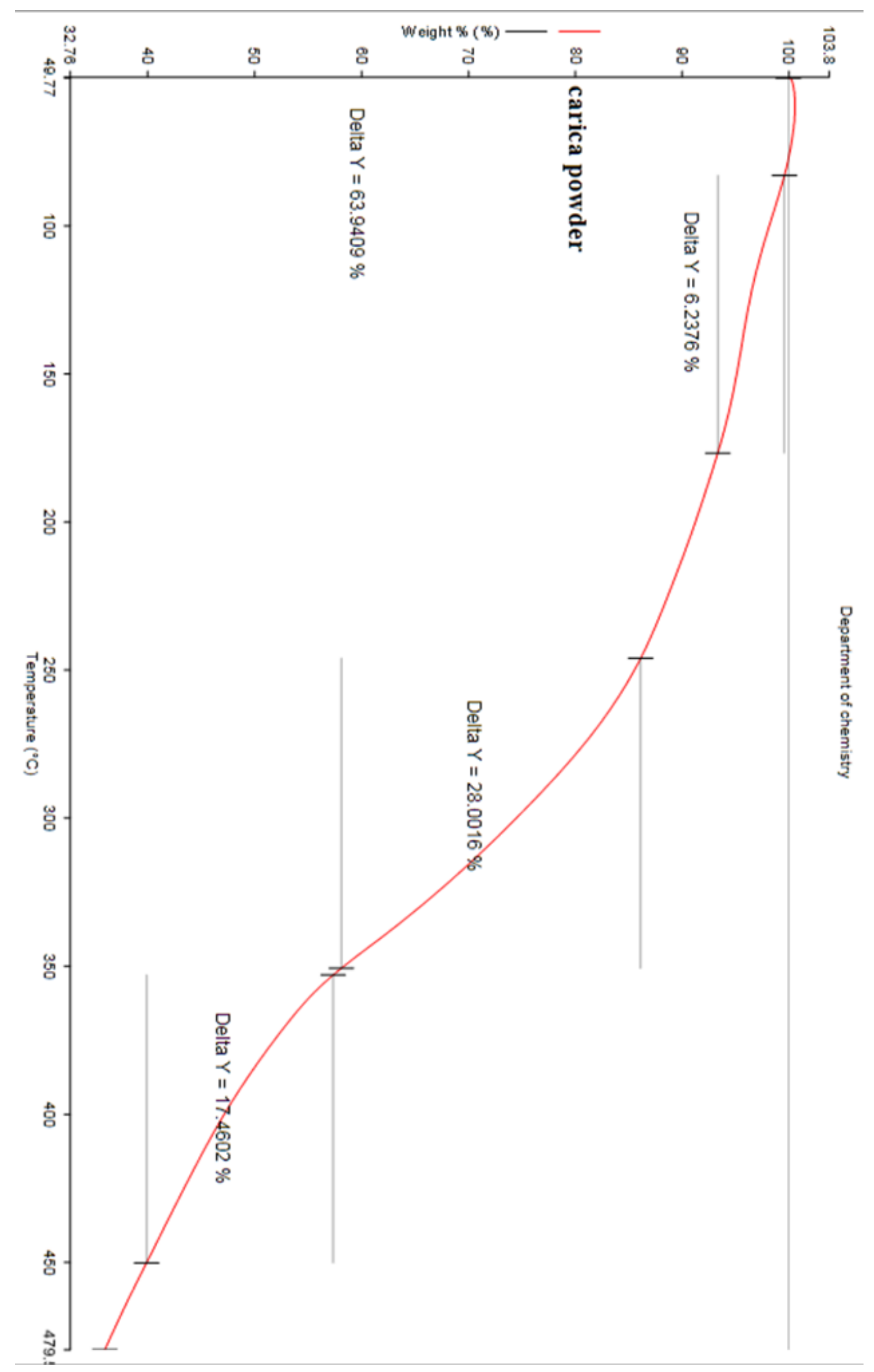

Figure 3. TGA Thermogram of the leaves of C. papaya. 


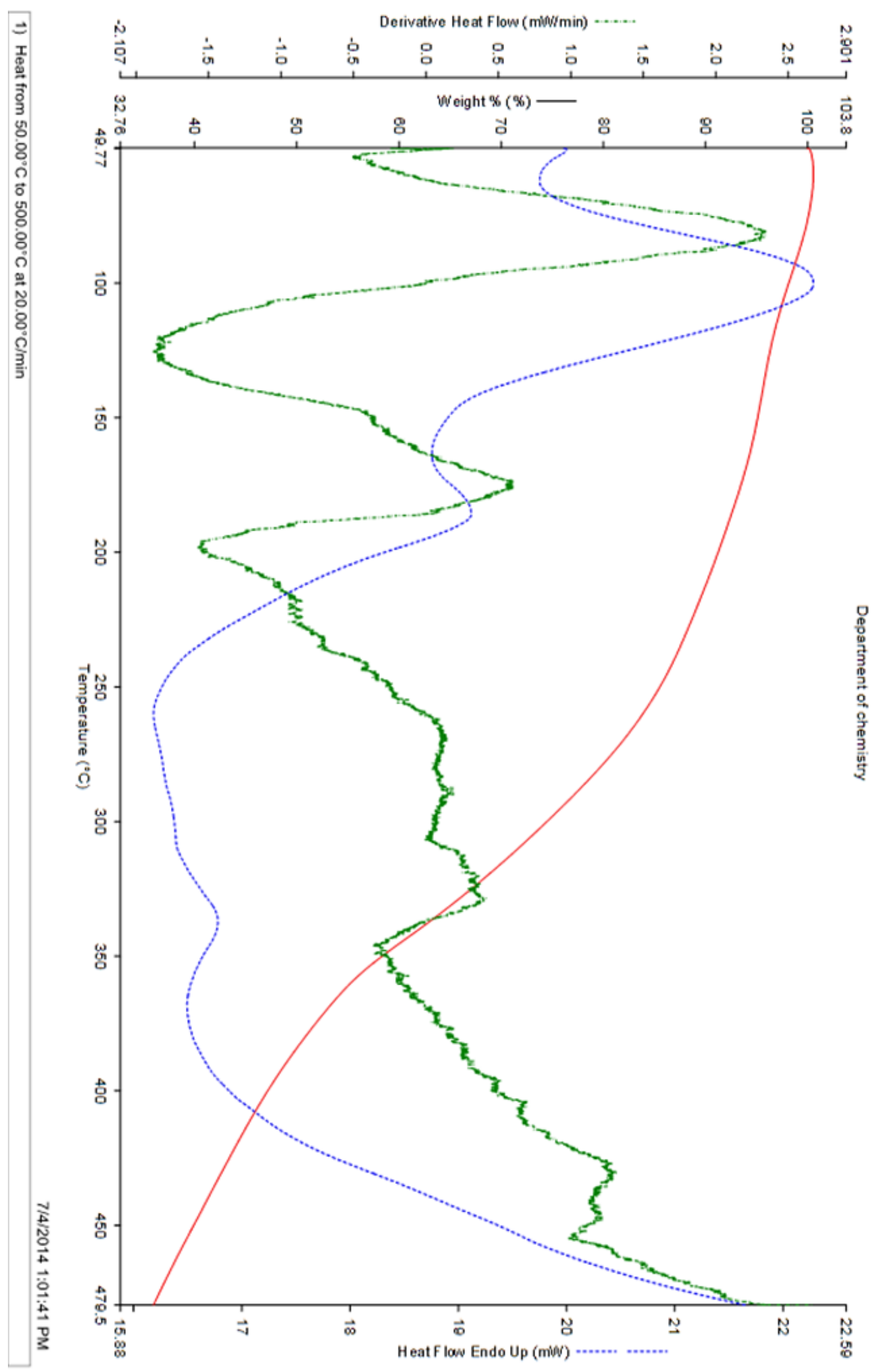

Figure 4. Total Thermogram of the leaves of C. papaya. 


\section{CONCLUSIONS}

The Thermal analysis method is a powerful tool for study of the effects of reaction atmosphere on thermal chemical characteristics for plant leaves sample. In Thermogravimetric analyzer (TGA), Differential Thermal Analyzer (DTA) and Differential Scanning Calorimetric (DSC) analysis results show that in DSC curve, Endothermic peak at $101{ }^{\circ} \mathrm{C}$ is attributed to dehydration/Water loss from surface and pores of the powder sample. Step at $215{ }^{\circ} \mathrm{C}$ is associated with second order phase transition such as Glass Transition and it should be further confirmed in second heating (During heat- cool- heat cycle). Endothermic peak at $336{ }^{\circ} \mathrm{C}$ is associated protease thermal decomposition /Beta Cyclodextrin breakdown. In the TGA Curve, the initial $4 \%$ weight loss is due to water loss from surface/pores of powder sample. Second weight loss between $200-450{ }^{\circ} \mathrm{C}$ is associated to degradation of cellulose and hemicellulose. In further analysis there is possibility of degradation of lignin between $450-800{ }^{\circ} \mathrm{C}$ by further extending the analysis temperature.

\section{Acknowledgement}

The authors are thankful for facilities provided by Center for excellence in chemical science and technology, Department of Chemistry, KSKV Kachchh University, Bhuj-Kutch-370 001, India.

\section{References}

[1] Mutalik G. S., Background paper No. SEA/RPD/Tradmed/RSG Meet 1/2, WHO office, N. Delhi (1972).

[2] Kirtikar K.R., Basu B.D., Indian Medicinal plants Vol. I and II, Lalit Mohan Basu and Co., Calcutta (1935).

[3] Chopra R. N., Chopra I.C., Handa K. L., Kapoor L. D., Indigenous Drugs of India, U.N. Dhur and Sons, Culcutta (1958).

[4] Nadkarni A. K., Indian Materia Medica Vol. I and II, Popular Book Depot, Bombay (1954).

[5] Mello V.J., Gomes M.T., Lemos F.O., Delfino J.L., Andrade S.P., Lopes M.T., et al. The gastric ulcer protective and healing role of cysteine proteinases from Carica candamarcensis. Phytomedicine 15 (2008) 237-244.

[6] Noriko Otsuki, Nam H. Dang, Emi Kumagai, Akira Kondo, Satoshi Iwata, Chikao Morimoto, Aqueous extract of Carica papaya leaves exhibits anti-tumor activity and immunomodulatory effects. J Ethnopharmacology 127 (2010) 760-767

[7] Fakeye T.O., Abu T., Adebisi O., A survey of the use of herbs among patients attending secondary health care facilities in Southwestern Nigeria. J Herbal Pharmacotherapy 2007 (In press). 
[8] Oyelola O., Evaluation of the hypoglycemic activity of Treculia Africana Decne (root) in normal and diabetic rats. M. Pharm (Clinical Pharmacy) Dissertation University of Ibadan Nigeria, 2005.

[9] Suhas J. Vyas, Taslim T. Khatri, Vijay R. Ram, Pragnesh N. Dave, Hitendra S. Joshi, Biochemical constituents in leaf of Carica papaya - ethnomedicinal plant of Kachchh region. International Letters of Natural Sciences 7 (2014) 16-20.

[10] Pravin N. Ram, Vijay R. Ram, Taslimahemad T. Khatri, Suhas J. Vyas, Pragnesh N. Dave, XRF analysis of Carica papaya leaves of semi arid region of Kachchh. International Letters of Natural Sciences 19 (2014) 15-24. 